\title{
Stress in tungsten carbide-diamond like carbon multilayer coatings
}

\author{
B. R. Pujada ${ }^{\text {a) }}$ \\ Netherlands Institute for Metals Research, Mekelweg 2, 2600 GA Delft, The Netherlands \\ and Department of Materials Science and Engineering, Delft University of Technology, \\ Mekelweg 2, 2628 CD Delft, The Netherlands \\ F. D. Tichelaar \\ Kavli Institute of Nanoscience, National Centre for HTEM, Delft University of Technology, Lorentzweg 1, \\ 2628 CJ Delft, The Netherlands \\ G. C. A. M. Janssen \\ Department of Materials Science and Engineering, Delft University of Technology, Mekelweg 2, 2628 CD \\ Delft, The Netherlands
}

(Received 7 July 2006; accepted 5 December 2006; published online 10 January 2007)

\begin{abstract}
Tungsten carbide-diamond like carbon (WC-DLC) multilayer coatings have been prepared by sputter deposition from a tungsten-carbide target and periodic switching on and off of the reactive acetylene gas flow. The stress in the resulting WC-DLC multilayers has been studied by substrate curvature. Periodicity and microstructure have been studied by transmission electron microscopy. It has been observed that compressive stress in the multilayers decreases when the bilayer thickness is reduced. Results show a minimum compressive stress for bilayer thickness of around $5 \mathrm{~nm}$. This behavior is discussed in terms of interface stress and mixing between layers. (C) 2007 American Institute of Physics. [DOI: 10.1063/1.2430905]
\end{abstract}

Nanocomposite hard coatings, consisting of metallic elements in a diamond like carbon matrix, are gaining considerable attention because of their potential use as protective coatings for a wide range of applications. These coatings exhibit very attractive tribological and mechanical properties such as low friction coefficient and high wear resistance in comparison to conventional ones. ${ }^{1-3}$ Protective coatings are usually under stress, which can contribute to mechanical failure. Therefore, it is imperative to control the stress in order to avoid delamination or cracking in hard coatings.

Stress in multilayer films composed of two alternating layers is of great interest because the presence of a high number of interfaces. Although the underlying theory for stress in multilayer films is not well understood, various authors have shown that the variation in stress can be the result of interaction with the substrate, interaction between each layer, and/or presence of interfaces. ${ }^{4-10}$ In the present letter we present and analyze results for compressive stress in a series of tungsten carbide-diamond like carbon (WC-DLC) multilayer coatings prepared by reactive rf magnetron sputtering. We present evidence of minimum compressive stress for bilayer thickness of $5 \mathrm{~nm}$ and an unusual large interface stress in these samples.

WC-DLC multilayer coatings have been produced via unbalanced magnetron deposition in a ATC-1500 F sputtering system (AJA Int.). These coatings were deposited by reactive rf magnetron sputtering from a WC target in an argon/ acetylene plasma onto a $100 \mathrm{~mm}$ silicon wafer with (100) orientation. Before deposition the chamber was evacuated to $1 \times 10^{-5} \mathrm{~Pa}$ and the wafer substrate was heated to a temperature of $160{ }^{\circ} \mathrm{C}$ for $90 \mathrm{~min}$. During coating deposition the substrate temperature was kept at $160{ }^{\circ} \mathrm{C}$ and the rf power on the WC target at $148 \mathrm{~W}$. The diameter of the target is

\footnotetext{
a) Author to whom correspondence should be addressed; electronic mail: b.r.pujadabermudez@tudelft.nl
}

$5.04 \mathrm{~cm}$ and the distance from target to substrate is $11 \mathrm{~cm}$. The substrate was rotated uniformly at $10 \mathrm{rpm}$. In order to obtain the multilayer structure, 5 SCCM (SCCM denote cubic centimeter per minute at STP) of acetylene gas flow was time modulated by a square wave (acetylene flow was switched on and off repeatedly during the total deposition), whereas the argon gas flow was held constant at 35 SCCM. The period of the time modulation was varied between $8 \mathrm{~s}$ and $20 \mathrm{~min}$. The total deposition time was kept at $2 \mathrm{~h}$. Coatings with different numbers of layers and layer thicknesses were obtained. Coating thicknesses were measured by crosssectional scanning electron microscopy micrographs (not shown here). By dividing the total thickness of the coating by the number of bilayers we obtain the bilayer thickness.

Multilayer structure of the coatings was confirmed by low-angle x-ray diffraction (XRD). The measurements were performed with a Bruker-AXS D 5005 diffractometer with a diffracted beam graphite monochromator. $\mathrm{Cu} K \alpha$ radiation was used for all measurements. Figure 1 shows the lowangle XRD patterns of samples prepared at $30 \mathrm{~s}$ and $3 \mathrm{~min}$ of acetylene flow modulation and with bilayer thicknesses of 5 and $30 \mathrm{~nm}$, respectively. By decreasing the time modulation of the acetylene flow, the distances between peaks increase. The multilayer structure in the XRD patterns starts to disappear at modulation times less than $30 \mathrm{~s}$.

A more detailed study of the microstructure of WC-DLC multilayers as well as the individual layers can be obtained through cross-sectional transmission electron microscopy (TEM). TEM was performed on a CM30T Philips TEM operating at $300 \mathrm{kV}$, and a Tecnai F20ST/STEM equipped with a Gatan imaging filter. Figure 2 shows a bright field TEM (BFTEM) image of the multilayer produced at $5 \mathrm{~min}$ of acetylene flow modulation. A first analysis of Fig. 2 reveals two alternating well-defined layers composed of $\mathrm{W}-\mathrm{C}: \mathrm{H}$ and WC, obtained when acetylene flow is switched on and off, respectively. Since tungsten absorbs more electrons than car- 


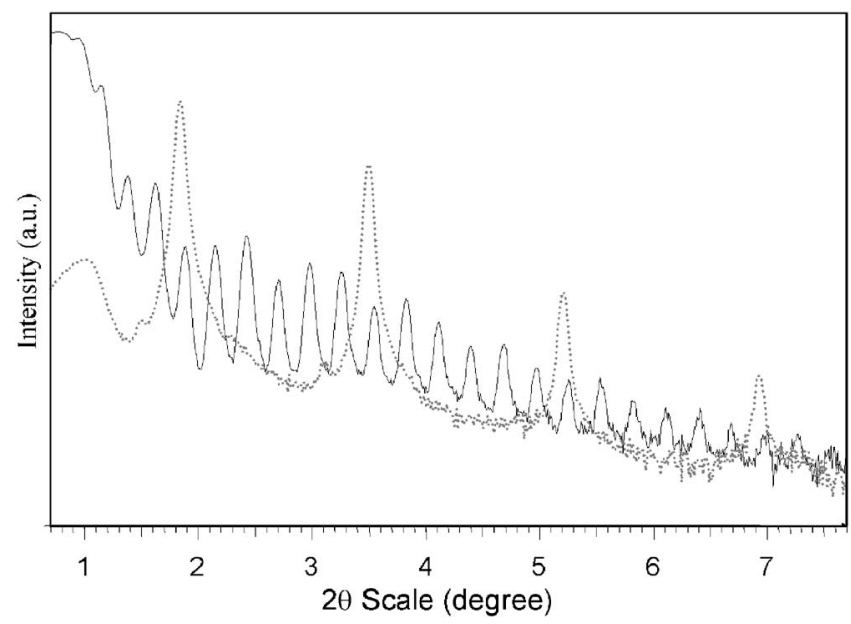

FIG. 1. Low-angle XRD patterns of WC-DLC multilayer coatings for $3 \mathrm{~min}$ (continuous) and $30 \mathrm{~s}$ (dashed) of acetylene flow modulation.

bon, it appears dark in the BFTEM image. Therefore, judging by the intensity difference in the layers, the $\mathrm{W}-\mathrm{C}: \mathrm{H}$ layer is characterized by a layer rich in carbon $(\mathrm{C})$, whereas the WC layer has much less C. From the BFTEM image we found a bilayer period of $50.7 \pm 0.5 \mathrm{~nm}$, and $\mathrm{WC}$ and $\mathrm{W}-\mathrm{C}: \mathrm{H}$ layer thicknesses of $17.4 \pm 0.5$ and $31.2 \pm 0.5 \mathrm{~nm}$, respectively. The inset in Fig. 2 shows narrow third and fourth layers of a very $\mathrm{C}$ rich composition above the WC layer of $1.1 \pm 0.1 \mathrm{~nm}$, and above this, a more or less broken up $\mathrm{W}$ rich layer of approximately $1 \mathrm{~nm}$.

The internal stress of the WC-DLC multilayer coatings was evaluated using the substrate curvature method. For this purpose, the curvature of the wafer was measured before and after deposition by applying two reflected parallel laser beams and the internal stress was calculated using the Stoney equation for stress measurement. ${ }^{11}$ Figure 3 shows the compressive stress of the WC-DLC multilayers as a function of the bilayer thickness. By decreasing the bilayer thickness from 103 to $5 \mathrm{~nm}$, the compressive stress decrease from -2.5 to $-0.8 \mathrm{GPa}$. At a bilayer thickness less than $5 \mathrm{~nm}$, the compressive stress increases rapidly up to $-1.5 \mathrm{GPa}$, a value close to the compressive stress in $\mathrm{W}-\mathrm{C}: \mathrm{H}$ coatings deposited using a continuous flow of 2.5 SCCM of acetylene gas $(-1.6 \mathrm{GPa}){ }^{12}$

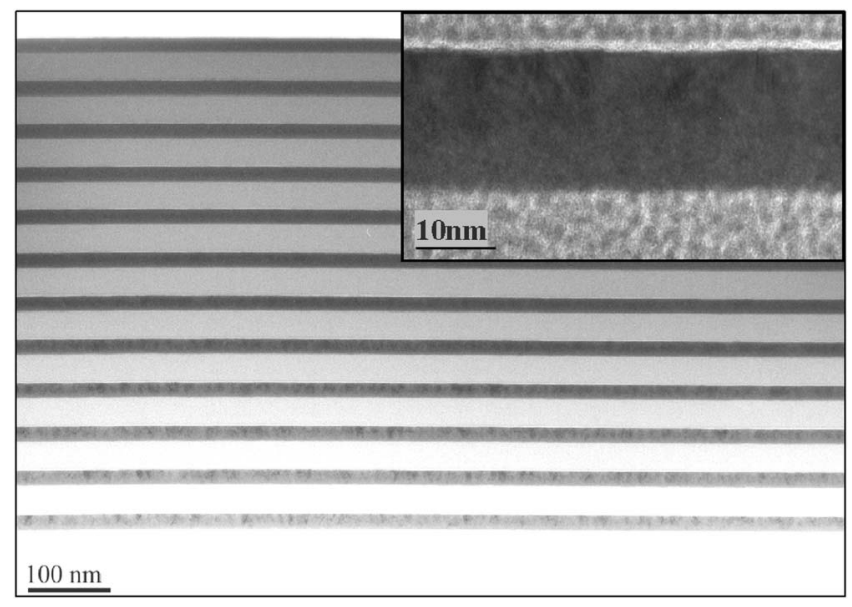

FIG. 2. Cross-sectional BFTEM image of WC-DLC multilayer coating obtained at $5 \mathrm{~min}$ of acetylene flow modulation. The higher magnification used for the inset shows a very thin carbon layer above the WC layer.

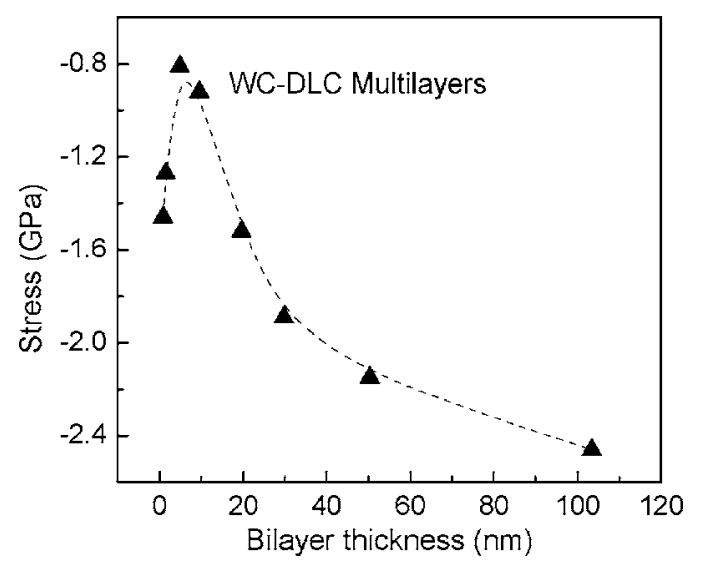

FIG. 3. Compressive stress as a function of the bilayer thickness in WCDLC multilayer coatings. A minimum value for stress is observed at around $5 \mathrm{~nm}$. The dashed line is a guide to the eyes.

Since that interface plays an important role in the total stress behavior in multilayer films, then consider the case of $N$ bilayers $A / B$, where $A=\mathrm{WC}$ and $B=\mathrm{W}-\mathrm{C}: \mathrm{H}$, with stresses $\sigma_{A}$ and $\sigma_{B}$ and thicknesses $d_{A}$ and $d_{B}$, respectively. The stress in a multilayer can be modeled as the thicknessaveraged stress in the individual layers plus the number of interfaces times the interface stress,

$$
\sigma_{\mathrm{sc}} d=N\left(\sigma_{A} d_{A}+\sigma_{B} d_{B}\right)+2 N f .
$$

By definition $\lambda=d / N$, where $d$ is the total thickness of the multilayer coating. This allows Eq. (1) to be written as

$$
\sigma_{\mathrm{sc}}=\frac{d_{A}}{\lambda} \sigma_{A}+\frac{d_{B}}{\lambda} \sigma_{B}+\frac{2 f}{\lambda} .
$$

For our series of depositions $d_{A} / \lambda$ and $d_{B} / \lambda$ were determined from the TEM micrographs. Also we ascertained that $\sigma_{A}$ does not depend on $d_{A}$, nor does $\sigma_{B}$ depend on $d_{B}$ : We measured stress in a separate series of films consisting of individual layers $A$ or $B$ with thicknesses ranging from 16 to $850 \mathrm{~nm}$. Of course the error increased with decreasing thickness but no systematical dependence of stress on individual layer thickness was observed. We found values for stresses of $\sigma_{A}=-4.56 \mathrm{GPa}$ and $\sigma_{B}=-1.53 \mathrm{GPa}$. Therefore the first two terms on the right in Eq. (2) are constants. Now we are able to determine $f$ from a plot of $\sigma_{\mathrm{sc}}$ vs $1 / \lambda$ (Fig. 4).

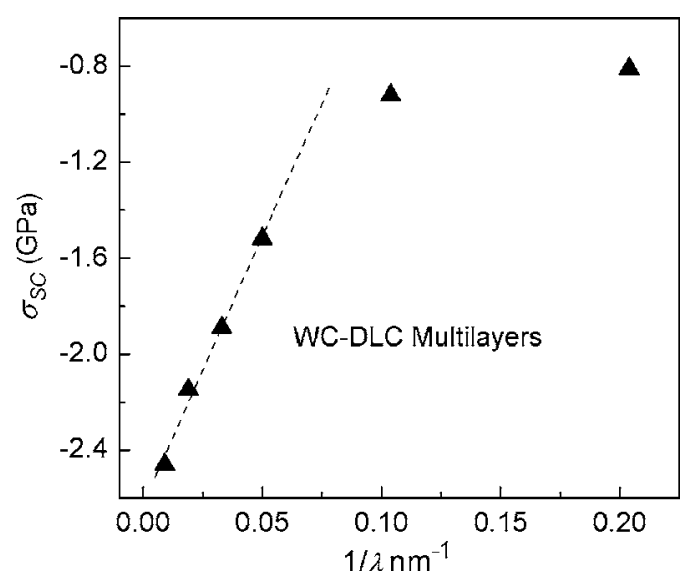

FIG. 4. Substrate curvature stress as a function of the inverse of bilayer thickness in WC-DLC multilayer coatings. The dashed line is a fit using Eq. (2) 

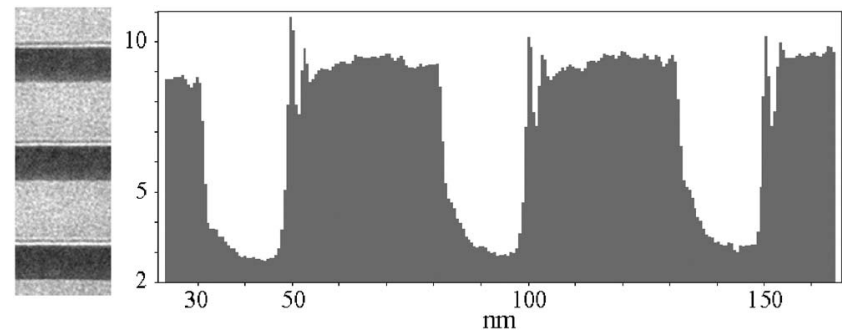

FIG. 5. EFTEM image of a part of WC-DLC multilayer coating obtained at $5 \mathrm{~min}$ of acetylene flow modulation. An energy window of $25 \mathrm{eV}$ at the $\mathrm{C}$ $K$-edge was used for imaging the carbon distribution. The intensity (arbitrary units) profile was obtained by averaging the intensity in the image in the direction parallel to the interfaces.

In Fig. 4 is shown the substrate curvature stress as a function of the inverse of the bilayer thickness in WC-DLC multilayer coatings. A linear stress behavior is observed for coatings with bilayer thickness of $20 \mathrm{~nm}$ and larger, in line with Eq. (2). Deviations from Eq. (2) are observed for coatings with bilayer thickness of $10 \mathrm{~nm}$ and smaller. This deviation is attributed to the mixing between layers, which is relatively more important for thin bilayers than for thick bilayers. Using Eq. (2) for samples with bilayer thickness of $20 \mathrm{~nm}$ and larger, we found an interface stress of $11.12 \mathrm{~N} / \mathrm{m}$. This value is extremely large for an interface stress.

In order to better understand the large interface stress, an energy filtered TEM (EFTEM) image of the multilayer coating produced at 5 min of acetylene flow modulation was taken with a $25 \mathrm{eV}$ energy window centred at $285 \mathrm{eV}$ close to the $\mathrm{C} K$-edge at $288 \mathrm{eV}$. Figure 5 shows part of the EFTEM image and the background corrected intensity profile of the EFTEM image. Firstly, from Fig. 5 it can be seen that the carbon concentration in the WC layer is not uniform; it is due to the remaining acetylene gas into the chamber after the acetylene flow is switched off, producing therefore an intermixing between layers. Also, the light stripe above the WC layer rich in $\mathrm{W}$ shows the enhanced carbon concentration at this location as observed before by BFTEM. Above the $\sim 1 \mathrm{~nm}$ thin carbon rich layer a thin $\mathrm{W}$ rich layer of $\sim 1 \mathrm{~nm}$ wide is also resolved. The carbon rich layer is attributed to the high quantity of acetylene gas that is going into the chamber when the acetylene flow is switched on. If we consider both very thin layers as an extra layer with constant thickness, the high interface tensile stress can alternatively be explained by stress in this layer. The combined $\mathrm{W}$ and $\mathrm{C}$ layer of $2 \mathrm{~nm}$ thickness has to have a stress of $5.5 \mathrm{GPa}$ in order to arrive at the same curvature as caused by an interface stress of $11 \mathrm{~N} / \mathrm{m}$. Such a stress can be arrived at by constrained shrinkage as proposed by Klokholm and Berry. ${ }^{13}$ In this model it is assumed that the material is deposited disordered. Subsequent ordering leads to shrinkage, hence tensile stress. Following this idea we arrive at a volume shrinkage of $1 \%-2 \%{ }^{14}$

In conclusion, we have studied compressive stresses in WC-DLC multilayer coatings. We have found that by decreasing the individual layer thickness, the compressive stress decreases, showing a minimum at $5 \mathrm{~nm}$ of bilayer thickness. The increase of compressive stress observed for bilayer thicknesses below $5 \mathrm{~nm}$ is explained by mixing. By the application of Eq. (2) we have found a large interface stress of $11.12 \mathrm{~N} / \mathrm{m}$. TEM micrographs of the WC-DLC coatings revealed the presence of a very thin carbon and tungsten layers. An alternative explanation for the interface stress in the form of stress in those layers is offered.

This research was carried out under Project No. MC7.02142B within the framework of the strategic Research program of the Netherlands Institute for Metals Research. X-ray diffraction was performed by N. M. van der Pers of the Department of Materials Science and Engineering (TU-Delft).

${ }^{1}$ C. Strondl, G. J. van der Kolk, T. Hurkmans, W. Fleischer, T. Trinh, N. M. Carvalho, and J. Th. M. De Hosson, Surf. Coat. Technol. 142-144, 707 (2001).

${ }^{2}$ A. Czyzniewski and W. Precht, J. Mater. Process. Technol. 157-158, 274 (2004).

${ }^{3}$ Y. T. Pei, D. Galvan, and J. Th. M. De Hosson, Acta Mater. 53, 4505 (2005).

${ }^{4}$ J. A. Ruud, A. Witvrouw, and F. Spaepen, J. Appl. Phys. 74, 2517 (1993).

${ }^{5}$ R. C. Cammarata, Prog. Surf. Sci. 46, 1 (1994).

${ }^{6}$ A. L. Shull and F. Spaepen, J. Appl. Phys. 80, 6243 (1996).

${ }^{7}$ S. Fayeulle and M. Nastasi, J. Appl. Phys. 81, 6703 (1997).

${ }^{8}$ R. C. Cammarata, K. Sieradzki, and F. Spaepen, J. Appl. Phys. 87, 1227 (2000).

${ }^{9}$ F. Spaepen, Acta Mater. 48, 31 (2000).

${ }^{10}$ X. Zhang and A. Misra, J. Appl. Phys. 96, 7173 (2004).

${ }^{11}$ P. A. Flinn, D. S. Garder, and W. D. Nix, IEEE Trans. Electron Devices 34, 689 (1987).

${ }^{12}$ B. R. Pujada and G. C. A. M. Janssen, Surf. Coat. Technol. 201, 4284 (2006).

${ }^{13}$ E. Klokholm and B. S. Berry, J. Electrochem. Soc. 115, 823 (1968).

${ }^{14}$ G. L. Leusink, T. G. M. Oosterlaken, G. C. A. M. Janssen, and S. Radelaar, J. Appl. Phys. 74, 3899 (1993). 